\title{
Investigation and treatment of constrictive pericarditis
}

\author{
M. K. A. D A Y E M, F. M. W A S F I, H . H. B EN T A L L, \\ J . F. GOO D W IN, AN D W. P. C L E L A N D \\ From the Departments of Medicine (Clinical Cardiology) and Surgery (Cardiothoracic Unit), Royal \\ Postgraduate Medical School, and Hammersmith Hospital
}

\begin{abstract}
Twenty-six patients with constrictive pericarditis have been investigated and treated-by surgical in addition to medical methods in 22 , and by medical management alone in four. A tuberculous aetiology was proved in only two patients but was probable in 10 others. One patient developed constriction of the heart following a stab wound, which caused a haemopericardium, and one after organization of a pericardial cyst. In the remainder no cause was found. The clinical features and diagnostic criteria are described, with emphasis on cardiac catheterization and angiocardiography. Operative results are given: two patients died after the operation, and the results are compared with other series. All surviving patients were improved: greatly in 13 and moderately in three. Four patients had a poor result due to long-standing myocardial disorder, and so we stress the importance of early operation, with reference to illustrative cases. Conversely, the practicability of treating certain patients by medical means alone is demonstrated. The criteria for operation are discussed with reference to repeated cardiac catheterization and angiocardiography.
\end{abstract}

Constrictive pericarditis is not a common disease. Wood (1961) described 40 cases; only 53 cases were seen at the Massachusetts General Hospital between 1914 and 1947 (Paul, Castleman, and White, 1948), and Burwell (1957) had studied only 66 patients with this disease up to 1953. This rarity may be due in part to the decreasing incidence of human tuberculous infection in the Western world in recent years, although constrictive pericarditis is common in South Africa (Schrire, 1959). Even with improved methods of diagnosis in recent years the disease appears still to be rare in Europe and North America.

Twenty-six patients with constrictive pericarditis were seen at Hammersmith Hospital between 1948 and 1965. Here we present their salient clinical and physiological features and analyse the roles of medical and surgical treatment in the management of this disease.

\section{SUBJECTS AND METHODS}

In our 26 patients the diagnosis was confirmed at operation in 22 and at necropsy in one. In addition to clinical examination, all patients had the appropriate radiographs and electrocardiograms; the plasma proteins, plasma electrolytes, and the blood urea were estimated, and a full blood coun $\stackrel{0}{\bar{\beta}}$ was made. A thorough search for evidence of present or past tuberculous infection included. in every patient, sputum and urine cultures, and Mantoux test utilizing a $1 / 100$ to $1 / 10,000$ con $\frac{\text { O }}{N}$ centration of P.P.D. Cultures of pleural or peri cardial fluids were made whenever possible, anक phonocardiography was performed in 12 patients

Right heart catheterization was carried out ing 16 patients. The right atrial, right ventricular, and pulmonary arterial pressure pulses were analysed음 The cardiac output, arteriovenous oxygen differ $\rightarrow$ ence, pulmonary capillary wedge and intra-arteria? pressures were recorded whenever possible. Tho response of the right atrial and wedge pressures to inspiration and the effect of the Valsalva manœuvre and respiration were also noted.

Angiocardiography by the venous or the righe atrial route was performed in seven patients using $85 \%$ hypaque (Preger, Dayem, Goodwin, anc Steiner, 1965).

\section{RESULTS}

AGE AND SEX The average age in this series wa 37 years, with a range from 9 to 70 years. Ove $\mathbb{8}$ half the patients were over 30 years of age. The male: female sex ratio was $1 \cdot 5: 1$. 
AETIOLOGY Tubercle bacilli were isolated from the pericardium in only two patients in this series. In a further 10 patients there was some evidence of a past tuberculous infection either from the histological examination of the excised pericardium or from a positive Mantoux test. In one patient the rapid onset of the signs and symptoms of constriction and the positive serological tests all made a viral aetiology probable. One patient had a pericardial cyst which had ruptured, and one developed constriction after a stab wound of the chest. No cause for the constrictive pericarditis could be detected in the remaining 12 patients. However, we assumed a tuberculous cause for the purposes of treatment in all patients, while realizing that other causes may have been operating.

HISTORY In 13 patients the duration of symptoms was one year or less prior to hospital admission. Nine gave a history of between one and 11 years. In the remaining four the duration of the disease could not be determined.

Figure 1 gives a summary of the main symptoms in the 26 patients. Dyspnoea was the commonest finding and was present in 18 patients. Cough, chest pain, pericardial and pleural effusion, and fever were less frequently encountered.

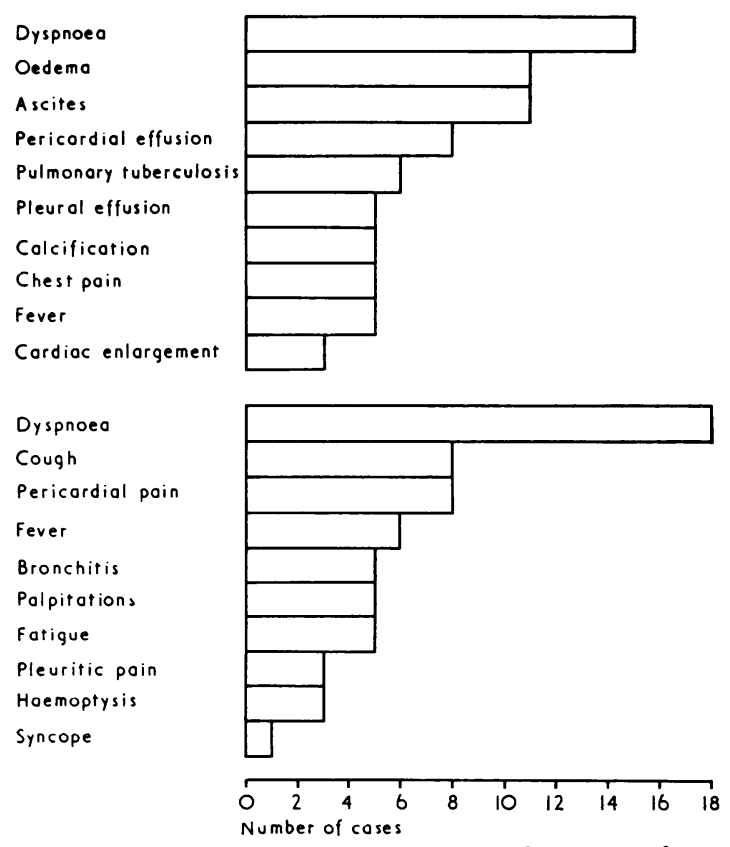

FIG. 1. Histograms showing the relative frequency of past and present symptoms and other features.
CLINICAL EXAMINATION Figures 2 and 3 show the frequency of the general and cardiac signs found in the 26 patients. The commonest sign was an early third sound, which was heard in 24 patients. A raised jugular venous pressure and hepatic enlargement were present in 21 and 20 patients respectively. The jugular venous pulse was of the usual type in this disease, with sharp ' $y$ ' descent and prominent ' $a$ ' and ' $v$ ' waves (Fig. 4). An increase in the height of the pressure on inspiration was common. Pulsus paradoxus and a quiet apex beat were present in just over half the patients.

Ascites preceded oedema in only eight patients, ascites alone was present in two, while oedema alone was present in five: both were absent in seven patients.

The blood pressure was always within the normal range, though the pulse volume was small clinically in 14 patients. Peripheral cyanosis was observed in 11 patients and clubbing of the fingers was found in three.

Atrial fibrillation was found in five patients, and atrial flutter in one. Wide splitting of the second heart sound (Beck, Schrire, and Vogelpoel, 1962) was detected in seven of our patients.

TUBERCULOUS INFECTION A striking feature was the complete absence of any evidence of present or past infection with tubercle bacilli in 14 patients. Only two had tubercle bacilli isolated from the pericardium. In a further seven the only evidence of past tuberculous infection was a positive Mantoux test.

BLOOD PICTURE AND CHEMISTRY The haemoglobin content of the blood was below $13.0 \mathrm{~g} . / 100 \mathrm{ml}$. in eight patients $(14.0 \mathrm{~g} . / 100 \mathrm{ml} .=100 \%$ in our laboratory). The erythrocyte sedimentation rate was above $15 \mathrm{~mm}$. in the first hour in only five patients. In spite of the frequency of hepatomegaly only six patients showed a reduced plasma protein level and an albumin:globulin ratio of 1.0 or less.

PHONOCARDIOGRAPHY Analysis of the 12 recorded phonocardiograms showed the presence of an early third heart sound in 11 patients (Fig. 5). The average interval between the aortic component of the second sound and the third sound was $0 \cdot 12$ second.

ELECTROCARDIOGRAPHY The electrical axis of the heart in the frontal plane was $+60^{\circ}$ or more in two-thirds of the patients. Right axis deviation of more than $+90^{\circ}$ was detected in four patients. Left axis deviation was not found. 
Raised J.V.P.

Enlarged liver

Pulsus parodoxus

Small pulse volume

Kussmaul's sign

Oedemo

Ascites

Pleural effusion

Peripheral cyanosis

Tachycardia

Atrial fibrlllation

Clubbing

Spleen

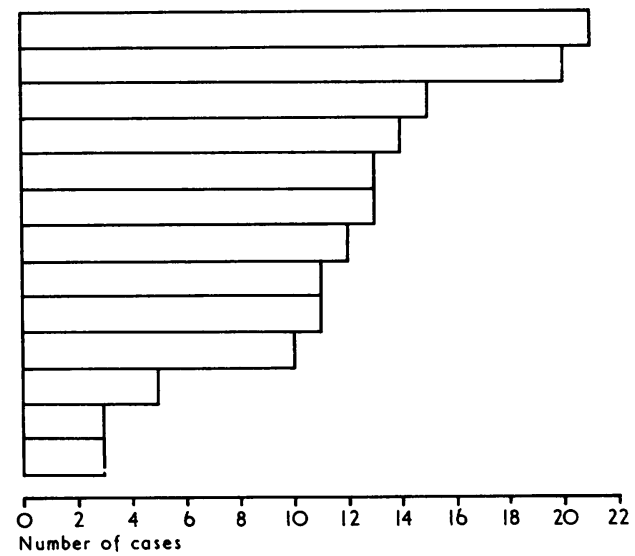

FIG. 2. Histogram showing the fre quency of the various physical signs. $J V P=j u g u l a r$ venous pressure ; Kuss maul's sign=increase in jugular $\overrightarrow{\mathrm{\omega}}$ venous pressure on inspiration.

FIG. 3. Histogram showing the frequency of cardiac signs.

3rd heart sound Indefinite opex beat Faint heart sounds Systolic murmur Wide split 2 nd sound R.V. Impulse Rub Fixed split 2nd sound Systolic click 4 th heort sound
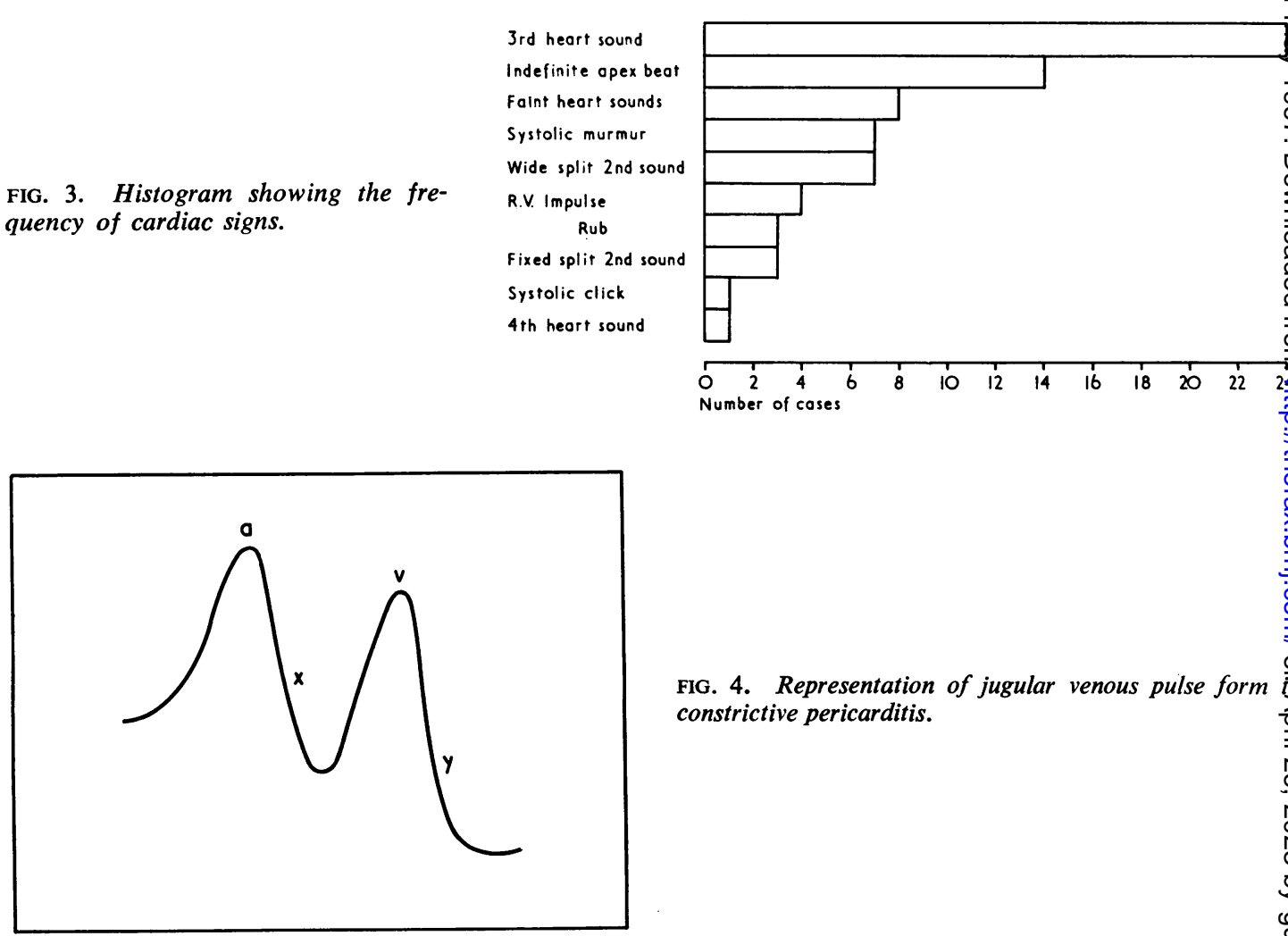

FIG. 4. Representation of jugular venous pulse form constrictive pericarditis.

FIG. 5. Phonocardiogram showing an early third heart sound (arrowed): A.A.=aortic area; L.F.=low frequency ; M.A.= mitral area.

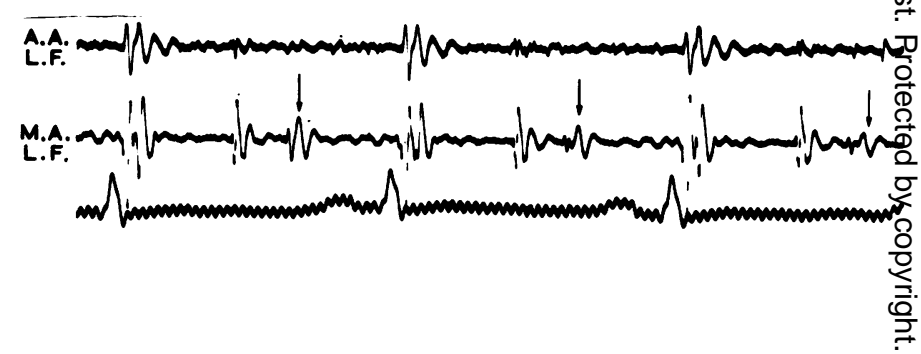



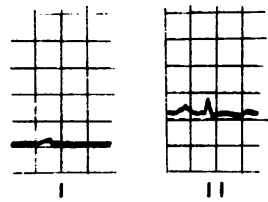

| 1

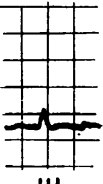

III
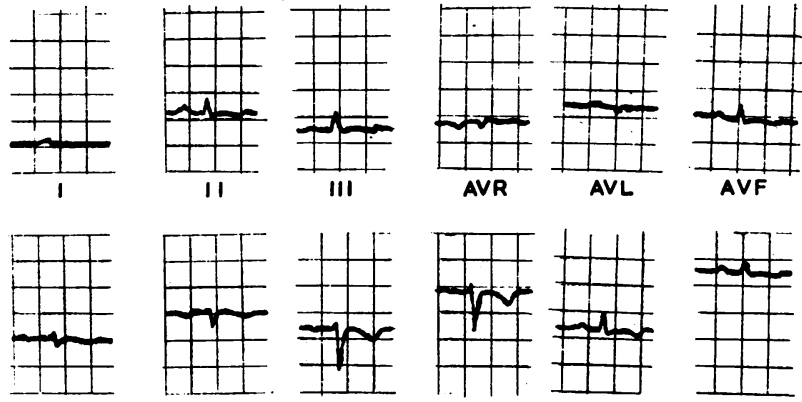

VI
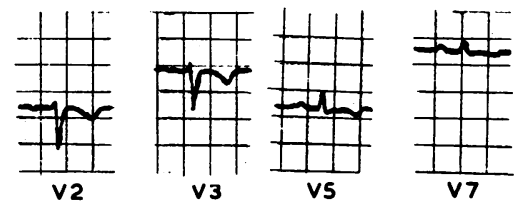

FIG. 6. Electrocardiogram of a patient with chronic constrictive pericarditis showing a notched $P$ wave, low voltage $Q R S$ complexes, inverted $T$ waves, and a dominant $R$ wave in lead $V_{4} R$.
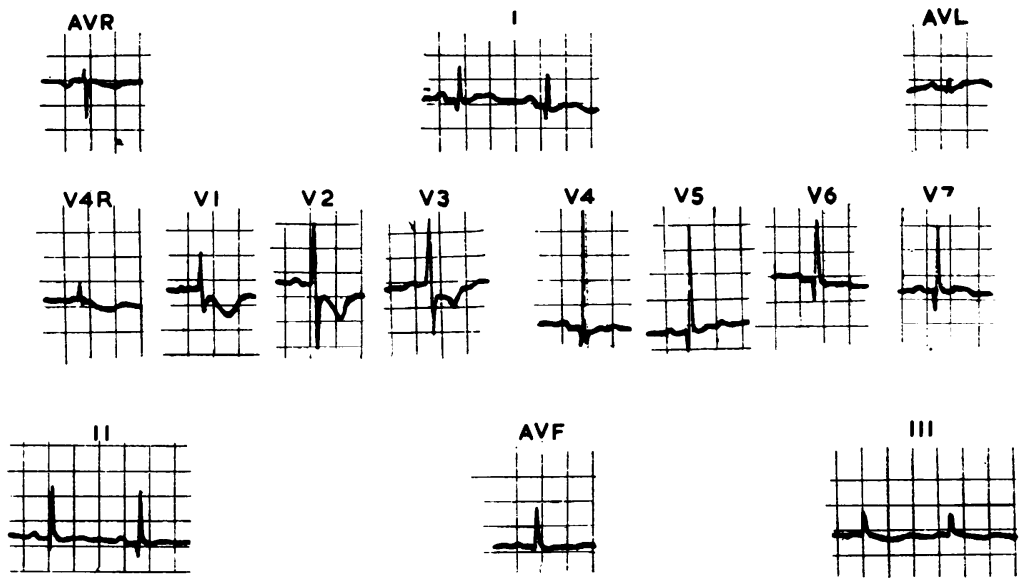

FIG. 7. Electrocardiogram in constrictive pericarditis of recent onset, showing inverted $T$ waves in $V_{1}$ to $V_{3}$, flat $T$ waves in $V_{4}$ to $V_{7}$, and tall $R$ waves in $V_{4}$ and $V_{5}(25$ and $20 \mathrm{~mm}$. respectively). There is left atrial enlargement, and a dominant $R$ in $V_{4} R$ and $V_{1}$.

The $\mathbf{P}$ wave was broad and notched in seven patients. Atrial fibrillation occurred in five patients and atrial flutter in one. Low voltage QRS complexes were seen in both the limb and chest leads in 15 patients (Fig. 6). Lead $V_{4} R$ showed a $Q R$ pattern in two patients and a dominant $R$ wave in another four. A short history was sometimes associated with tall $\mathbf{R}$ waves in the left chest leads (Fig. 7).

RADIOLOGY A summary of the appearances on plain films is given in Figure 8. Calcification of the pericardium (Figs 9a and $b$ ) was demonstrated in $16(60 \%)$ patients. This incidence is very similar to that observed in most other series (Wood, 1961; Hugh, 1962). The cardiothoracic ratio varied between 0.38 and 0.72 , the average being 0.51 .
FLUOROSCOPY In eight out of 10 patients fluoroscopy revealed diminished cardiac pulsations, more pronounced on the right side in six. Irregular and unequal cardiac pulsation is a useful diagnostic sign. A pleural effusion was observed in 12 patients.

ANGIOCARDIOGRAPHY There was thickening of the pericardium in the frontal and lateral projections on the right cardiac border (Goodwin, 1965) (Fig. 10 ). An analysis of the tracings of the angiograms of seven patients showed that the variation in the size of the left atrial shadow during the cardiac cycle was greatly reduced in four patients $(8.3 \%$ of the diastolic atrial volume) and within normal limits in three (39\% of the diastolic atrial volume). A normal change in volume of the left atrium during the cardiac cycle was found in only three 

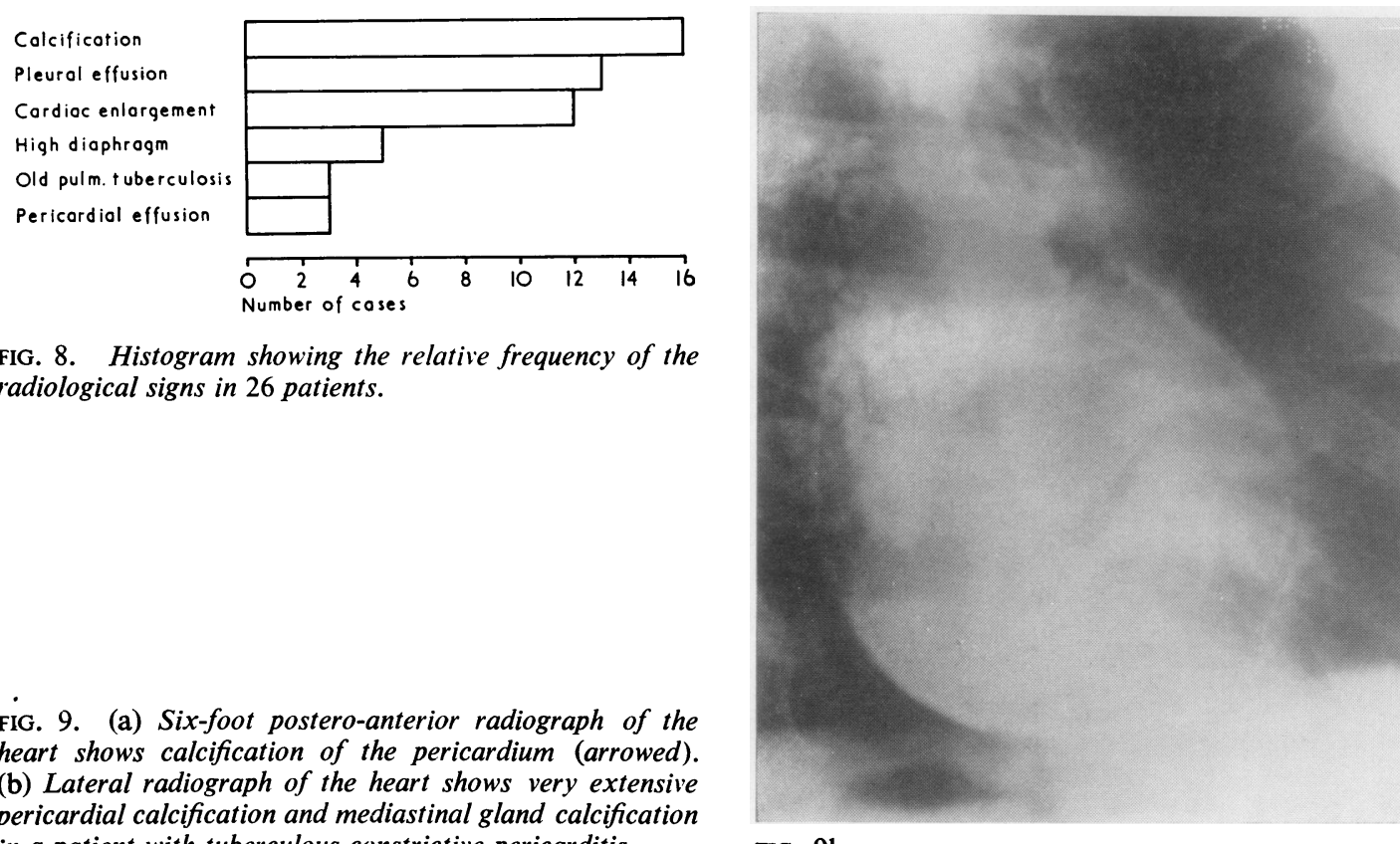

FIG. 8. Histogram showing the relative frequency of the radiological signs in 26 patients.

FiG. 9. (a) Six-foot postero-anterior radiograph of the heart shows calcification of the pericardium (arrowed). (b) Lateral radiograph of the heart shows very extensive pericardial calcification and mediastinal gland calcification in a patient with tuberculous constrictive pericarditis.

FIG. $9 b$

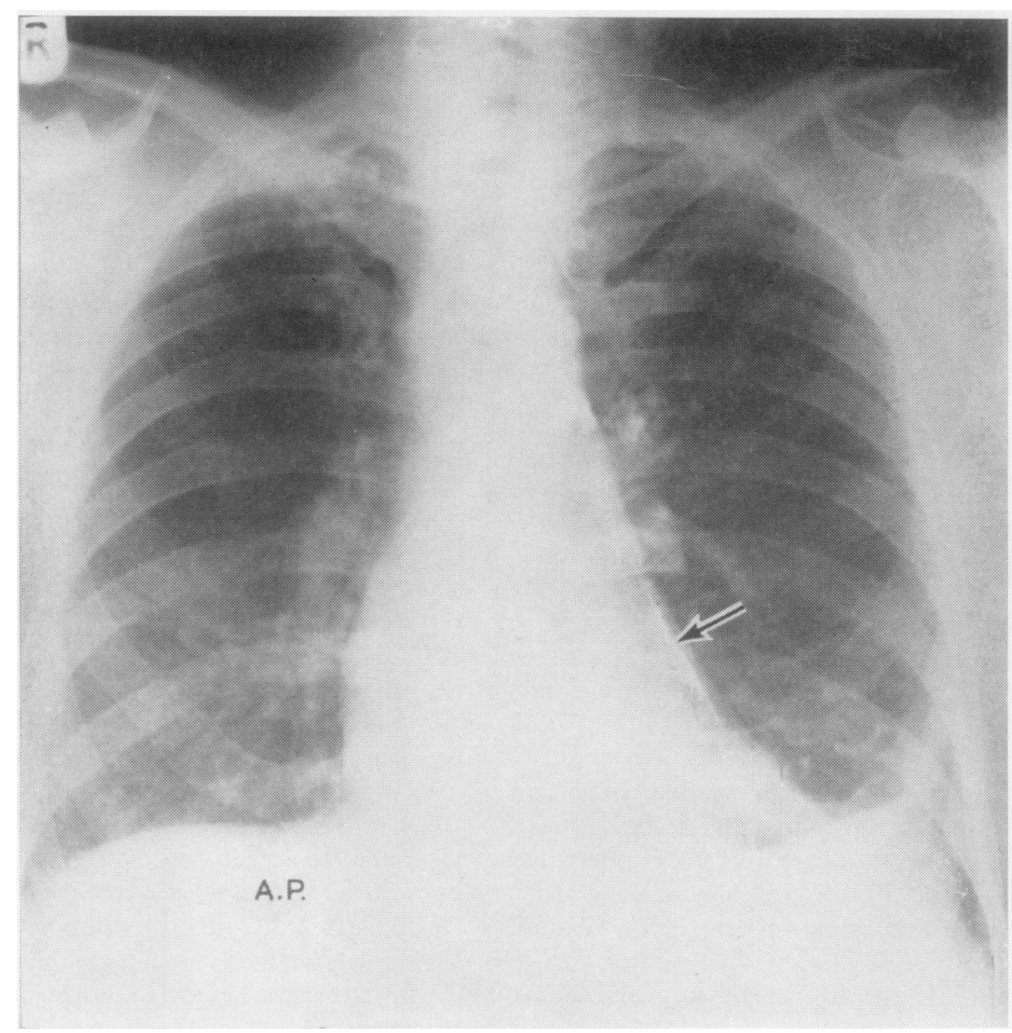

FIG. 9a 


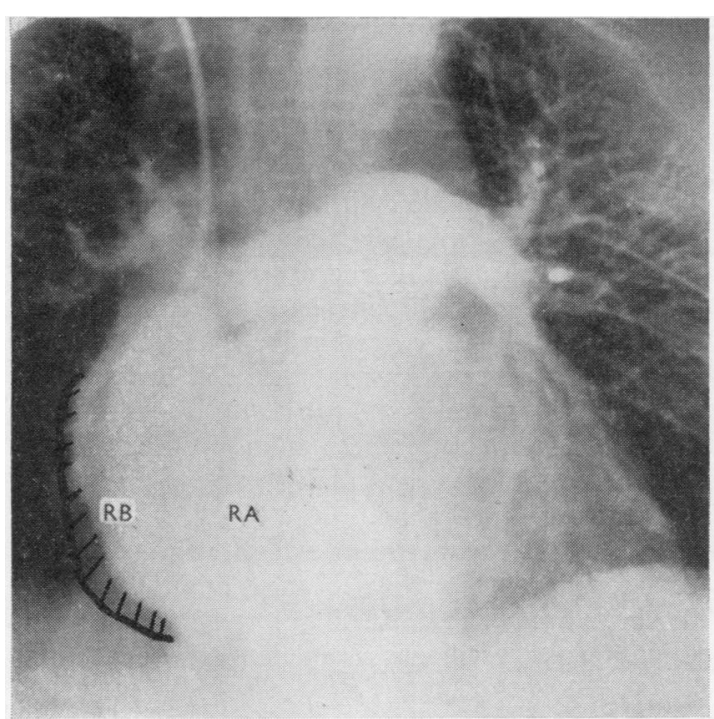

FIG. 10. Frontal projection of right atrial angiocardiogram showing thickened pericardium (hatched) between the contrast medium in the right atrium $(R . A$.) and the right atrial border (R.B.).

patients, who did not need surgery, suggesting that reduced volume change is associated with severe disease (Preger et al., 1965).

HAEMODYNAMICS Full details of the haemodynamic findings in the 16 patients catheterized are available from the authors. The mean right atrial pressure was $10.5 \mathrm{~mm}$. $\mathrm{Hg}$ (range $2-19 \mathrm{~mm}$. $\mathrm{Hg}$ ). A steep ' $\mathrm{y}$ ' descent was found in 12 patients (Fig. 11). An attempt was made to correlate the jugular venous pressure level above the sternal angle, as estimated on the bedside, with the right atrial pressure measured during catheterization. The correlation was poor and in most patients the venous pressure at the bedside was lower than that which would have been predicted from that recorded in the right atrium. A rise in the right atrial pressure with inspiration (Kussmaul's (1873) sign) was observed in eight patients.

The average right ventricular systolic pressure was $33 \mathrm{~mm}$. $\mathrm{Hg}$. The right ventricular pressure dropped rapidly in early diastole, but only rarely did it fall below zero. An early diastolic dip was found, however, in 12 patients and was followed by a rapid rise in the right ventricular pressure to a plateau which remained during the rest of the diastole. The end-diastolic pressure in the right ventricle was raised to a level varying between 5 and $22 \mathrm{~mm}$. $\mathrm{Hg}$, the average being $12.5 \mathrm{~mm}$. $\mathrm{Hg}$.
The mean pulmonary arterial pressure ranged from 13 to $30 \mathrm{~mm}$. $\mathrm{Hg}$ and the cardiac output between 2.9 and 6.2 litres/minute. The average indirect left atrial wedge (PCP) pressure was $14 \mathrm{~mm}$. Hg.

The Valsalva manœuvre resulted in an abnormal response in two patients. This may be due to the fact that the positive intrathoracic pressure is readily transmitted to the right atrial cavity because of the interference caused by the calcified pericardial barrier. The increase in the venous-right atrial pressure gradient thus produced augments the venous inflow to the right atrium during the strain period.
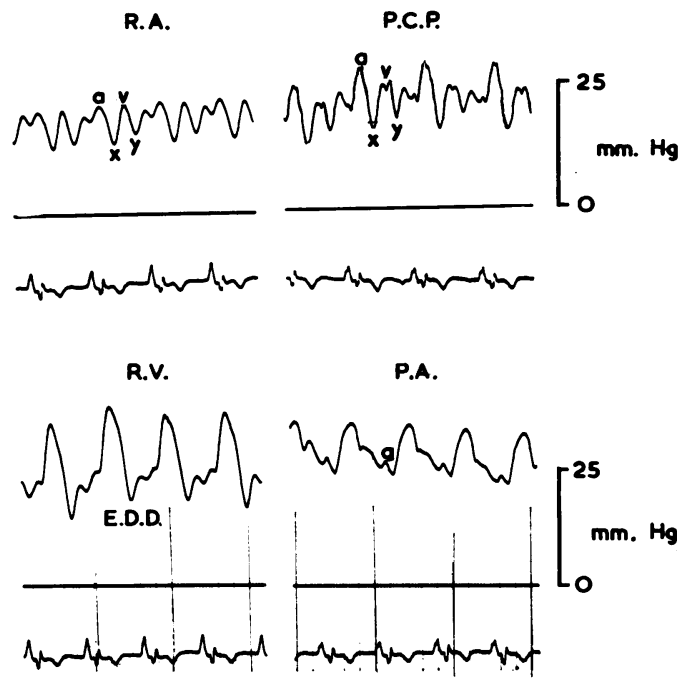

FIG. 11. Haemodynamic findings in a patient with chronic constrictive pericarditis showing rapid ' $x$ ' and ' $y$ ' descents in both the right atrial (R.A.) and pulmonary capillary wedge pressure pulse (P.C.P.). There is an early diastolic dip (E.D.D.) followed by a plateau in the right ventricular pressure pulse (R.V.) as well as a prominent atrial contraction wave $(a)$ in the pulmonary artery pressure pulse (P.A.). Note the tall $P$ waves in the electrocardiogram (lead II), indicating right atrial enlargement.

Exact superimposition of the pulmonary artery and right ventricular pressure pulses was possible in four patients. In all of them the pressure in the right atrium during a trial systole (the ' $a$ ' wave) was higher than the pulmonary diastolic pressure. The pressure in the pulmonary artery rose either simultaneously with, or $0.02 \mathrm{sec}$. before, the rise of the pressure in the right ventricle. Thus atrial contraction alone was able to open the pulmonary valve and the isometric contraction period was abolished. 
OPERATIVE RESULTS Twenty-two patients were operated upon and two died. In one (P. H., aged 9 years) a thickened pericardial sac was found at operation, trapping a flabby myocardium. Despite good relief of constriction his blood pressure fell and a second thoracotomy was performed on the same day because of suspected intrathoracic bleeding. Cardiac arrest occurred on opening the chest, again in the process of closing it, and he died four hours after the operation. The other (B. W., aged 59 years) had glomerulonephritis and Henoch-Schönlein purpura in addition to constrictive pericarditis. A successful operation was followed by improvement for nine months. Then frequent attacks of chest pain culminated in acute myocardial infarction and death 10 months after the operation. Necropsy confirmed the extensive nature of the infarction and showed calcification of both coronary arteries and thrombosis of the right main artery.

The operation was attended with few complications. A tear in the right atrium during the process of decortication was the commonest and occurred in four instances. In all, however, the tear was sutured either directly or with a piece of pericardium overlying it, and gave rise to no further trouble. Left phrenic nerve palsy resulting in a raised left cupola of the diaphragm with paradoxical movement was observed in three patients.

Atrial fibrillation followed the operation in two patients who were previously in sinus rhythm. In one patient fibrillation occurred a few hours after the operation, in the other it started on the third post-operative day, and in both it has persisted since. Decortication was commenced on the left side and then continued on the right in order to avoid respiratory distress or pulmonary oedema which might be produced by releasing the right side before the left: after operation we did not observe any such complications.

In most patients it was possible to perform a fairly complete excision of the anterior half of the pericardial sac, and re-operation for persistent constriction was necessary in only two instances. However, sometimes the calcium penetrated so deeply into the myocardium that it was impossible to remove it. In these instances the remaining plaques rarely interfered with the general effectiveness of the decortication.

Follow-up of the 20 survivors showed that 13 had symptoms which included dyspnoea, cough, palpitations, fatigue, syncope, and bronchitis. None of the patients with atrial fibrillation reverted to sinus rhythm after the operation. The third heart sound was still heard after the opera- tion in 11 out of 20 patients, but it was delaye in time. A positive Kussmaul's sign, pulsus para doxus, and a small arterial pulse volume persisteg in four patients. Ascites and enlargement of the liver remained in three patients and peripher贵 cyanosis in two. Wide splitting of the second hearf sound remained in nearly all those in whom th was present before the operation.

Post-operative radiographs showed that the heart frequently increased in size following release of pericardial constriction (Fig. 12). Some areas of calcification were still observed in seven out of 20 patients, though less than pre-operativel A high diaphragm was noted in five patients andp a pleural effusion in two.

An attempt was made to evaluate the results of the operation according to the condition of the 20 survivors (Table I). The condition of the patients was classified as 'excellent' if all the signts

T A B LE I

बै

RESULTS OF SURGERY IN CONSTRICTIVE PERICARDITS (Present Series)

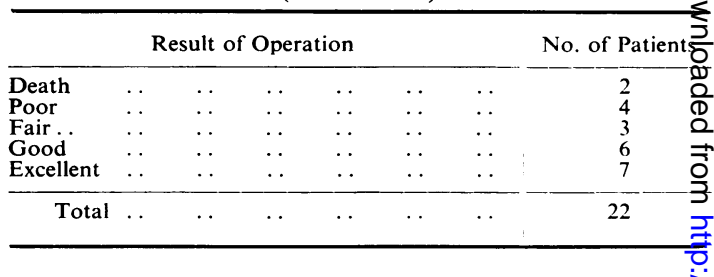

and symptoms of the disease disappeared afte요 the operation; as 'good' if only one of these remained; as 'fair' if two or three of the sign and symptoms persisted; and as 'poor' if mosif. or all of them were still observed after the decortication.

Four patients were still incapacitated after tho operation and were classified as 'poor'. In on of these the cause proved to be incomplete. decortication, and a second operation with removate of the remaining pericardial thickening proved successful. The second failure was due to a comp bination of residual pericardial constriction and pleural fibrosis. Both were removed at a sup sequent operation, and the patient improved greatly. In the other two patients the cause was thought to be poor myocardial function.

Three patients were classified as 'fair'. How ever, in spite of the persistence of some of thi signs and symptoms of the disease, their cond tion was much better than before the operatio $\$$ and they managed a nearly normal life. The resul was 'good' in six patients and 'excellent' in nine. 


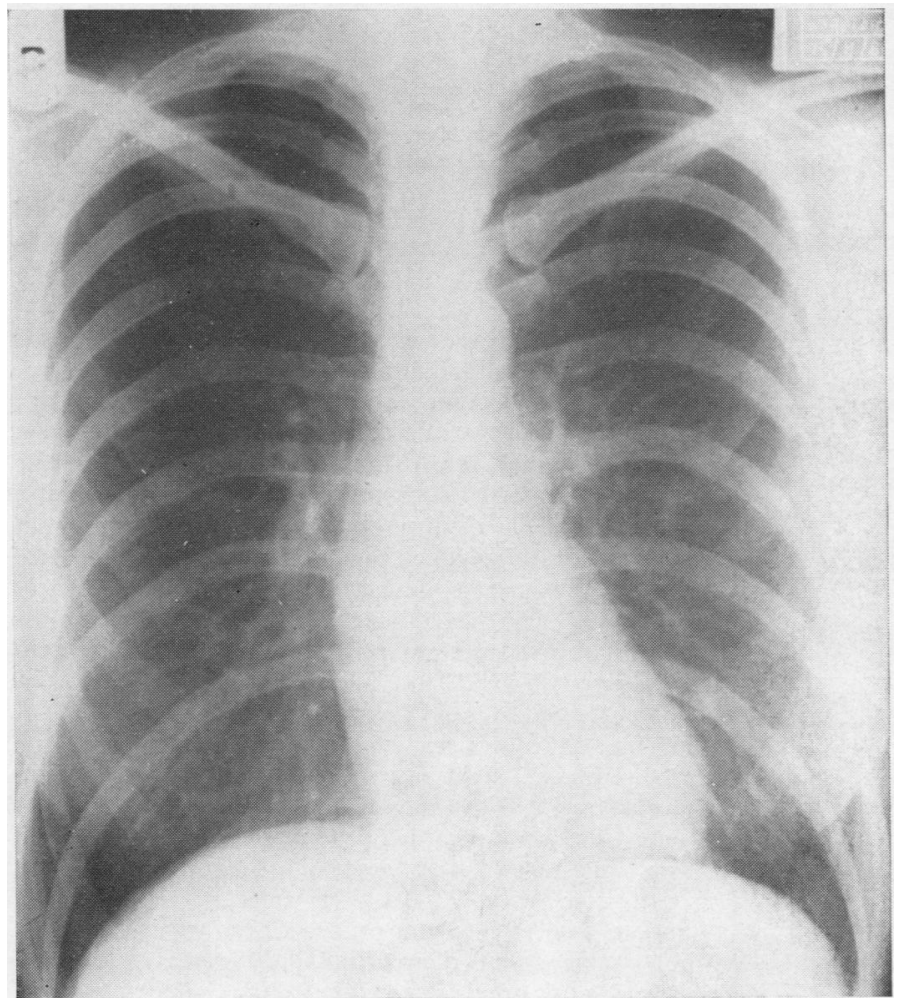

FIG. 12. (a) Six-foot postero-anterior pre-operative radiograph of the heart of a patient with chronic constrictive pericarditis. (b) Six-foot posteroanterior chest radiograph of the same patient shows an increase in heart size after successful pericardiectomy.

(a)

(b)

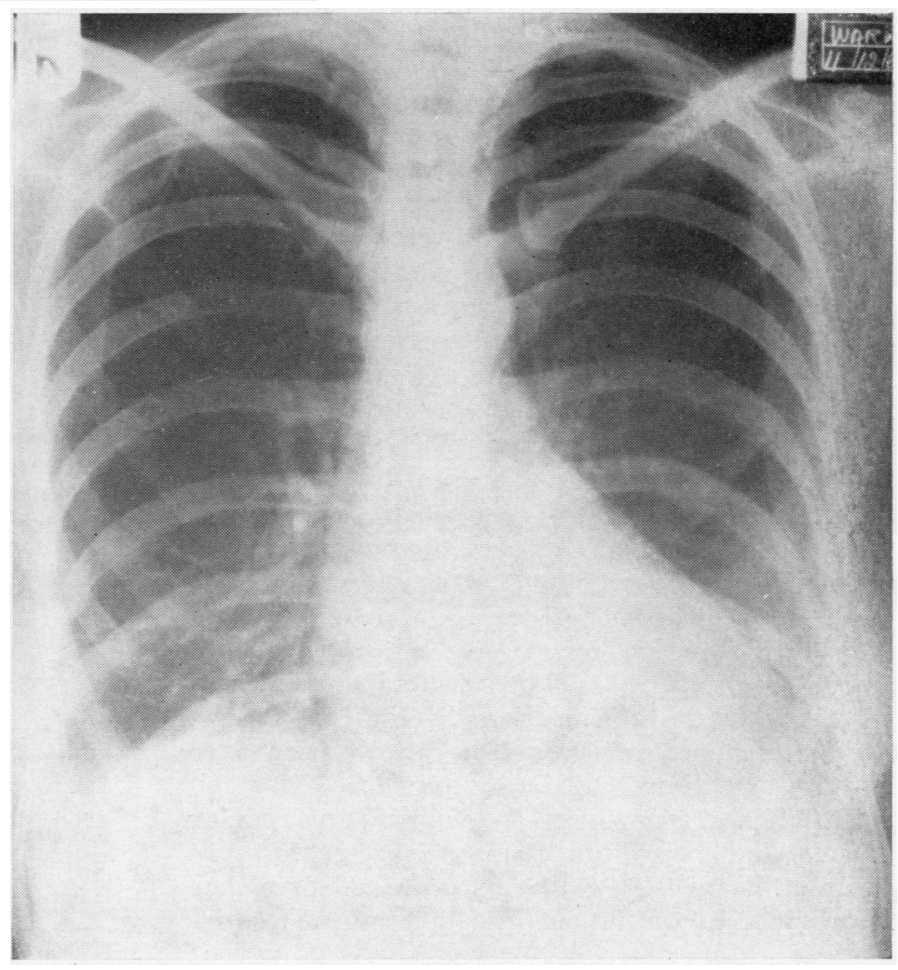




\section{DISCUSS:ON}

In this series $85 \%$ of patients were asymptomatic and leading normal lives in the follow-up period of from 19 years to eight months (mean $7 \cdot 2$ years).

The results of operation for constrictive pericarditis vary a great deal. The operative and postoperative mortality in the recorded series since 1952 ranged between $3 \%$ and $45 \%$ (Table II). The majority of these patients maintained considerable improvement for many years. The largest series with the lowest mortality is that of Portal, Besterman, Chambers, Holmes Sellors, and Somerville (1966).

T A B LE II

RESULTS OF SURGERY FOR CONSTRICTIVE PERICARDITIS (Published Series)

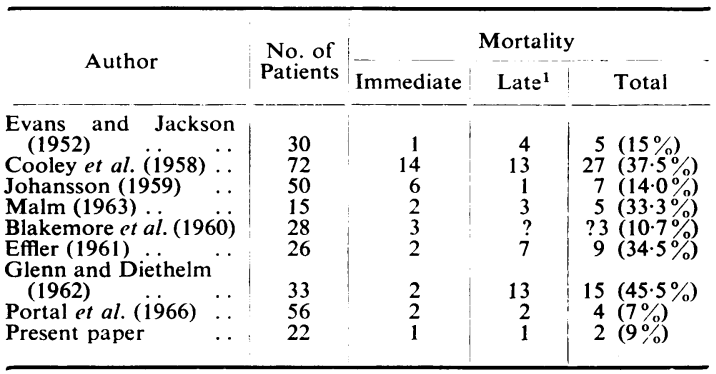

${ }^{1}$ Not all late deaths were due to the effects of the pericarditis

In the 22 operations performed at Hammersmith Hospital for constrictive pericarditis there was one operative death. One other patient died, 10 months after surgery, of an acute myocardial infarction which was proved at necropsy. We believe that these results were partly due to the policy of advising surgery wherever possible before the pathological process had involved the myocardium and partly by meticulous care in removal of all the constricting pericardial tissue.

The importance of early operation cannot be over-estimated. If the pathological process is left for too long complications are apt to occur. Thus fibrosis may extend into the myocardium, destroying and replacing the heart muscle. Dines, Edwards, and Burchell (1958) have shown that there is myocardial atrophy in patients dying from long-standing constrictive pericarditis. This atrophy is uniform and not limited to the areas subjacent to the constriction. Several patients in our series have been found at operation to have calcium extending into the myocardium. This calcification and fibrosis partly replaces the cardiac muscle, and in addition it may interfere with the contractility of the existing myocardial fibres. The importance of the myocardial factor in determin- ing the outcome of the disease has been stresse舫 by Malm (1963), Cooley, Clagett, and Kirkliq (1958), Burwell (1957), and Harvey, Ferrer, Cath cart, Richards, and Cournand (1953). In two ofour patients poor myocardial function resulted i only moderate improvement after operation despite a good pericardiectomy. Their case histories are given to illustrate this point.

CASE 1 N. R. was a woman aged 52 years. She waş admitted to hospital with six months' history of pro gressive ankle oedema, ascites, and dyspnoea. Ther $\vec{x}$ was no history of tuberculosis. Clinical examination showed a jugular venous pressure $+14 \mathrm{~cm}$. above the sternal angle with a marked ' $y$ ' descent. The pulsis was irregular in rhythm and small in volume, witt obvious paradox. The cardiac impulse was hypoo dynamic, the heart sounds were muffled, and an early third sound was heard at the apex. Examination of the chest showed a right pleural effusion. The electrom cardiogram showed low voltage $T$ waves. Radio graphy showed a large heart and pericardial calcificaeo tion. The Mantoux test was negative. Serum proteing were reduced, with a reversal of the albumin: globulin ratio. This was found to be due both tळ poor nutrition resulting from the low cardias output and to excessive protein loss from the gut. Aळ operation in May 1961 a thick mass of calcium was found to extend into the myocardium at several sites Most of the calcified and thickened pericardium was removed and good expansion of the ventricles was noted. The patient improved after the operation, bu? follow-up three years after the operation showed raised jugular venous pulse to $3-6 \mathrm{~cm}$. above the. sternal angle with a rapid ' $y$ ' descent and a thire heart sound. The second heart sound was widely splie and fixed.

CASE 2 C. J. J. was a man aged 43 years. In 1953 routine chest radiograph showed calcification of the pericardium. He was then admitted to hospital, wherê he was found to have a raised jugular venous pulse an early diastolic third heart sound, slight ankle oedema, and ascites. Right heart catheterizatiog showed a raised right atrial pressure (Table III). The electrocardiogram showed low voltage $T$ waven together with broad and notched $P$ waves. Pericardiectomy was performed in 1955 . Very extensive

T A B L E I I I

RESULTS OF RIGHT HEART CATHETERIZATION BEFORQ AND AFTER PERICARDIECTOMY (Case 2)

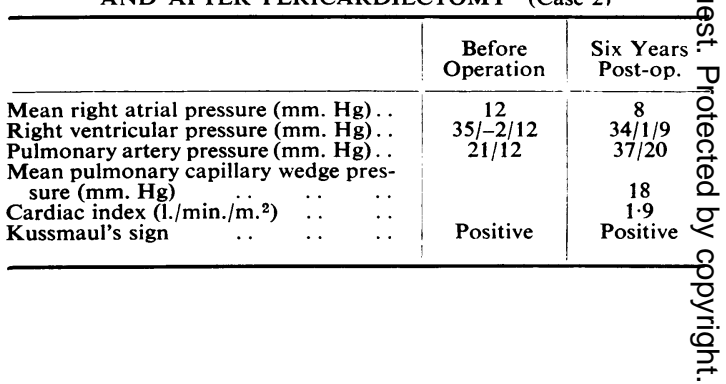


calcification of the pericardium was found involving the right atrium, the superior and inferior venae cavae, and the right ventricle. Pericardial compression was not so severe over the left ventricle. After the operation his symptoms were improved, but he still had grade I exercise intolerance with occasional palpitations, easy fatigability, a jugular venous pulse $5 \mathrm{~cm}$. above the sternal angle, and a positive Kussmaul sign. He also had slow atrial fibrillation. Radiography showed a slightly enlarged heart with pericardial calcification. Right heart catheterization was performed six years after the operation (Table III) and revealed a slight rise of the right atrial, wedge, and pulmonary artery pressures together with a low cardiac output. Angiocardiography showed only slight pericardial thickening.

In spite of the generally good operative results certain factors must be considered before advocating surgery. Signs and symptoms of constriction may be due, in the milder cases with recent history, to compression of the heart by the inflamed pericardium and pericardial effusion or caseating material before irreversible fibrosis and calcification have occurred. In these patients improvement may follow medical treatment, and in some patients operation may ultimately prove unnecessary. Owing to this, our policy has been to advise surgery in all long-standing and severe cases, but in less severe or acute patients only if the haemodynamic disability persists for more than three months despite adequate anti-tuberculous and supportive therapy. It is possible that an abnormal difference between the systolic and diastolic volumes of the left atrium measured angiographically may prove a useful indication that surgery will not be needed (Preger et al., 1965). Two of our patients improved on medical treatment. In one patient the aetiology was probably tuberculous (case 3 ) and in the other a viral aetiology was more probable (case 4). Their histories are given below.

CASE 3 D. D., a man aged 24 years, was admitted to Hammersmith Hospital in 1963 with four months' history of fever, cough, and loss of weight. He was found to have a jugular venous pressure $4 \mathrm{~cm}$. above the sternal angle, with a positive Kussmaul's sign. The cardiac apex beat was impalpable and there was an early diastolic dip in the right ventricle followed by a plateau. Angiocardiography showed a thickened pericardium. The patient was given streptomycin, isoniazid, and para-aminosalicylic acid and he soon started to improve. Follow-up one year afterwards showed that the jugular venous pressure was $+2 \mathrm{~cm}$. above the sternal angle, with a negative Kussmaul's sign. No third heart sound was heard and there was no arterial pulsus paradoxus. Right heart catheteriza-
T A B LE IV

RESULTS OF RIGHT HEART CATHETERIZATION BEFORE AND AFTER TREATMENT (Case 3)

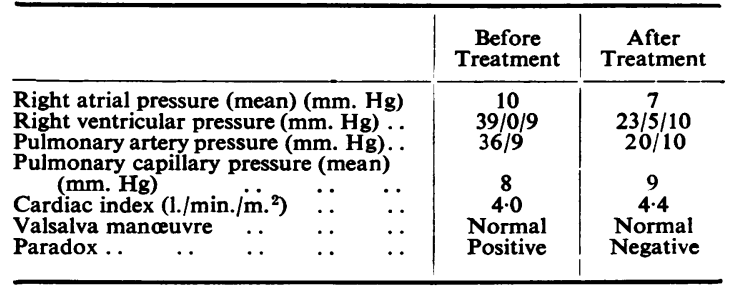

tion was repeated five months after the beginning of treatment. Results showed (Table IV) a drop in right atrial, right ventricular, and pulmonary artery pressures, disappearance of the 'dip-and-plateau' pattern in the right ventricular pressure pulse, little rise in the cardiac output, and absence of pulsus paradoxus. The patient was without symptoms, so we thought that operation was no longer necessary: he is being kept under observation, as the right ventricular enddiastolic pressure is still raised.

CASE 4 K. Z. was a man aged 30. His illness started in October 1962 with fever, dyspnoea, and chest pain on exertion. He was admitted to another hospital, where he was found to have a pericardial friction rub, enlargement of the heart, and a positive Mantoux test. The jugular venous pressure was elevated and there were cardiographic changes typical of pericarditis. He was then transferred to this hospital in December 1962 under the care of Dr. Arthur Hollman, where he was found to have a jugular venous pulse $14 \mathrm{~cm}$. above the sternal angle with a sharp ' $y$ ' descent. Auscultation revealed a loud early third sound at the apex and a split second sound which did not move on respiration. The liver was one finger below the costal margin. The electrocardiogram showed signs of chronic pericarditis. Right heart catheterization showed the right atrial, pulmonary capillary wedge, and right ventricular pressures to be elevated, and angiocardiography showed a thickened pericardium. No evidence of tuberculous infection was found, but viral studies showed evidence of recent infection with an adenovirus. He was given streptomycin, isoniazid, and para-aminosalicylic acid for 11 months. Since then his progress has been excellent. Follow-up in June 1963 showed that he was very well and without dyspnoea or chest pain. His jugular venous pressure was only $1 \mathrm{~cm}$. above the sternal angle and Kussmaul's sign was negative. His arterial pulse was normal and no paradox was detected. On auscultation no third heart sound was heard and his second heart sound was found to be normally split.

We are grateful to Professor R. E. Steiner and his colleagues in the Department of Radiodiagnosis for help with the radiological aspects of this work. Mr. Gerald Rainbow gave expert technical advice. 


\section{REFERENCES}

Beck, W., Schrire, V., and Vogelpoel, L. (1962). Splitting of the second heart sound in constrictive pericarditis, with observations on the mechanism of pulsus paradoxus. Amer. Heart J., 64, 765 .

Blakemore, W. S., Zinsser, H. F., Kirby, C. K., Whitaker, W. B., and Johnson, J. (1960). Pericardiectomy for relapsing pericarditis and chronic constrictive pericarditis. J. thorac. cardiovasc. Surg. $39,26$.

Burwell, C. S. (1957). Editorial. Constrictive pericarditis. Circulation, 15,161 .

Cooley, J. C., Clagett, O. T., and Kirklin, J. W. (1958). Surgical aspects of chronic constrictive pericarditis. A review of 72 operative cases. Ann. Surg., 147, 488.

Dines, D. E., Edwards, J. E., and Burchell, H. B. (1958). Myocardial atrophy in constrictive pericarditis. Proc. Mayo Clin., 33, 93.

Effler, D. B. (1961). Chronic constrictive pericarditis treated with pericardiectomy. Amer. J. Cardiol., 7, 62.

Evans, W., and Jackson, F. (1952). Constrictive pericarditis. Brit. Heart J., 14, 53.

Glenn, F., and Diethelm, A. G. (1962). Surgical treatment of constrictive pericarditis. Ann. Surg., 155, 883.

Goodwin, J. F. (1965). Bedside clues to the diagnosis of heart disease. Amer. J. Cardiol., 15, 81.
Harvey, R. M., Ferrer, M. I., Cathcart, R. T., Richards, D. W., and Cournand, A. (1953). Mechanical and myocardial factors in chronic constrictive pericarditis. Circulation, 8, 695.

Hugh, A. E. (1962). Radiographic features of constrictive peri carditis. Tubercle (Lond.), 43, 412.

Johansson, L. (1959). Constrictive pericarditis. Acta chir. scand., 117 104.

Kussmaul, A. (1873). Ueber schwielige Mediastino-Pericarditis und den paradoxen Puls. Berl. klin. Wschr., 10, 433, 445, and 461.

Malm, A. (1963). Chronic constrictive pericarditis with speciafs reference to pre- and post-operative hemodynamics. Dis. Chest $\overrightarrow{0}$
44,307 .

Paul, O., Castleman, B., and White, P. D. (1948). Chronic constrictive pericarditis: a study of 53 cases. Amer. J. med. Sci., 216, 361.

Portal, R. W., Besterman, E. M. M., Chambers, R. J., Holmes, Sellors, T., and Somerville, W. (1966). Prognosis after operation for constrictive pericarditis. Brit. med. J., 1, 563.

Preger, L., Dayem, M. K. A., Goodwin, J. F., and Steiner, R. E (1965). Angiocardiographic studies of pericardial disease. Lancet $\mathrm{N}$ 2, 701 .

Schrire, V. (1959). Experience with pericarditis at Groote Schuur Hospital, Cape Town: an analysis of one hundred and sixty cases studied over a six-year period. $S$. Afr. med. J., 33, 810. N

Wood, P. (1961). Chronic constrictive pericarditis. Amer. J. Cardiol.윽 